\title{
30. NOTAS PTERIDOLÓGICAS DE GALICIA. IV
}

\author{
Francisco X. SOÑORA
}

Palabras clave. Pteridófitos, corología, Galicia, España.

Hymenophyllum tunbrigense (L.) Sm.

A CORUÑA: Monfero, río Picheira, afluente del río Eume en la parte posterior del Embalse de A Capela. 29TNJ8302, 400 m.s.m., 02-IV-89. As Pontes, límites con Mañon, en las proximidades de la Provincia de Lugo, río de Solloso, afluente del río Sor, 29TPJ0022, 300 m.s.m., 30-VIII-89. Cerdido, cerca de Porto do Cabo, en un reguero que vierte en el río das Mestas. 29TNJ7730. 150 m.s.m., 08-IX-90. As Somozas, límites con Ortigueira, proximidades del río Mera, cerca de Pando. 29TNJ9123, 200 m.s.m., 08-IX-91. As Pontes, límites con Ortigueira, en el río Mera, cerca de Soutochao, 29TNJ9323, 250 m.s.m., 10-VIII-91.

LUGO: Ourol, río Besteburiz, afluente del río Landro, 29TPJ1324, 300 m.s.m., 22-IX-90. Ourol, límites con Viveiro, río de Santar, afluente del río Sor, 29TPJ0122, 300 m.s.m., 15-IX-91.

Son siete nuevas citas para Galicia que añadimos a las anteriores ya conocidas: Laínz M. (1968), Cremades y Bárbara (1987), Soñora y Ortiz (1988 y 1989). Siempre en el subsector Galaico Septentrional (sector Galaico-Asturiano) y en el seno de Blechno-Quercetum roboris R.Tx. y Oberdorfer, 1958. Generalmente rupícola sobre rocas de diferente naturaleza: granitos, serpentinas y esquistos; a veces epífito sobre restos de árboles caídos, pero siempre en laderas con exposición N o NW.

Puede concluírse que en el norte de Galicia es una especie común en su hábitat, en pequeñas poblaciones casi siempre puras y que rara vez cubren más de $0,25 \mathrm{~m} 2$, estando enormemente amenazadas por la destrucción de las fragas que son reemplazadas por repoblaciones con especies alóctonas, mayoritariamente eucalipto; alteración de cursos de agua e incendios forestales.

Vandenboschia speciosa (Willd.) Kunkel

A CORUNA: As Somozas, límites con Ortigueira, río Mera, cerca de Pando, 20TNJ9123, 200 m.s.m., 08-IX-91. Cariño, A Capelada, cerca del río Seixo de Landoi, 29TNJ8639, 150 m.s.m., 12-VI-91.

Nuevas citas para la provincia de A Coruña de este helecho considerado de interés continental, raro y vulnerable por los mismos motivos que los señalados para la especie anterior, ya que vive en hábitats similares. Indicado por Nyman para Galicia (Merino B., 1909) ha sido confirmada anteriormente su presencia en la provincia de A Coruña (Cremades y Barbara, 1987; Guitián P., Amigo y Guitián J., 1987). Añadir, además, que entre las varias poblaciones encontradas en As Somozas, una de ellas superaba los $45 \mathrm{~m} 2$ de extensión.

Culcita macrocarpa Presl.

A CORUNA: As Somozas, límites con Ortigueira, en un pequeño barranco sobre la orilla del río Mera, cerca de Pando, 29TNJ9123, 200 m.s.m., 08-IX-91.

Una sola población, pequeña, de una decena de ejemplares, bien desarrollados y fértiles. Tercera localidad gallega, equidistante de las dos anteriores (Laínz, 1968; Soñora y Ortiz 1989).

Thelypteris palustris Schott.

A CORUÑA: Valdoviño, «Fraga da lagoa de Pantín». 29TNJ7132, 0 m.s.m., 04-VII-84.

Helecho raro en Galicia, citado reiteradamente en Begonte, provincia de Lugo (Merino B., 1909; Allorge P., 1927; Bellot F. y Casaseca B., 1968). Está poco citado para el resto de Galicia: O Grove, provincia de Pontevedra (Merino B., 1909) y Santiso, provincia de A Coruña (Silva-Pando F.J. et al., 1987).

Huperzia selago (L.) Bernh. subsp. selago

A CORUNA: As Pontes, monte Caxado, 29TNJ9519, 757 m.s.m. 23-IV-89. 
LUGO: Viveiro, monte Castelo (Penido Gordo). 29TPJ1229, 527 m.s.m., 23-IV-89.

Confirmación de la primera cita gallega hecha por Merino: «Encontrada por el Sr. Rodríguez Franco en las faltas del Monte Castelo, próximo a la parroquia de Galdo» (Merino B., 1909). Unos pocos ejemplares, no en las faldas del monte, sino en la parte más alta. Señalada posteriormente en Os Ancares, provincia de Lugo (Reinoso J. y Rodríguez Oubiña J., 1987) y en A Capelada (Cedeira), provincia de A Coruña (Soñora F.X. y Ortiz S., 1988) y de nuevo encontrada en ésta última provincia.

Lycopodiella inundata (L.) J. Holub

LUGO: Muras, cerca de la carretera a Viveiró, 29TPJO815, 700 m.s.m., 06-X-90.

Nueva localidad para este licopodio citado ya en las cuatro provincias gallegas (Merino B., 1909; Allorge P., 1927; Silva Pando F.J. et al., 1987).

NOTA: Todos los pliegos de los táxones reseñados en este trabajo, se encuentran depositados en el herbario SANT.

\section{BIBLIOGRAFIA}

ALLORGE, P. -1927- Sur quelques plantes rares ou intéressantes de Galice. Bull. Soc. Bot. France, 74: 947-952. .

BELLOT, F. y B. CASASECA -1968- Notas sobre la Flora Gallega. Trab. Dep. Bot., Madrid, 1(1): 51-56.

CREMADES, J. y I. BARBARA -1987- Aportaciones a la pteridoflora gallega. Acta Bot. Malacitana, 12: 252-253.

GUITIÁN, P., J. AMIGO y J. GUITIÁN -1987- Flora Gallega VII Bol. Soc. Brot. ser. 2ª̣, LX: 139-146.

LAÍNZ, M. -1968- Aportaciones al conocimiento de la Flora Gallega, VI: 1-39. Madrid.

MERINO, B. -1909- Una nueva localidad de «Lycopodium inundatum L.» Bol. R. Soc. Esp. Hist. Nat., IX: 437-438.

MERINO, B. -1909-Sobre los helechos de Galicia. Bol. R. Soc. Esp. Hist. Nat., IX: 188-191.

MERINO, B. -1909- Flora descriptiva e ilustrada de Galicia III. Santiago de Compostela.

REINOSO, J. y RODRÍGUEZ OUBIÑA, J. -1987- Hallazgo de Huperzia selago (L.) Berhn. ex Schrack y Mart. subsp. selago en Galicia. Acta Bot. Malacitana, 12: 254.

SILVA PANDO, F.J., V. RODRÍGUEZ GRACIA, X.R. GARCÍA MARTÍNEZ y E. VALDÉS BERMEJO - 1987-Aportaciones a la Flora de Galicia II. Bol. Soc. Brot. ser $2^{a}$, LX: 29-68. SOÑRA F.X. y S. ORTIZ -1988- Notas pteridológicas de Galicia II. Acta Bot. Malacitana, 13: 374-375.

SONORA, F.X. y S. ORTIZ -1989- Notas pteridológicas de Galicia III. Acta Bot. Malacitana, 14: $258-259$.

(Aceptado para su publicación en Marzo de 1992)

Dirección del autor: Laboratorio de Botánica. Departamento de Bioloxía Vexetal. Facultade de Farmacia. Universidade de Santiago. 15706. Santiago de Compostela. 